\title{
INCIDENCIA DEL POLEN DE CHENOPODIACEAE- AMARANTHACEAE EN LA ATMÓSFERA DE MÁLAGA Y SU RELACIÓN CON LOS PARÁMETROS METEOROLÓGICOS
}

\author{
Marta RECIO, M. Mar TRIGO, F. Javier TORO y Baltasar CABEZUDO
}

\begin{abstract}
RESUMEN. Incidencia del polen de Chenopodiaceae-Amaranthaceae en la atmósfera de Málaga y su relación con los parámetros meteorológicos. En este trabajo se presentan los resultados obtenidos en Málaga por el tipo polínico Chenopodiaceae-Amaranthaceae, tras el muestreo aerobiológico realizado durante los años 1992-1997 por medio de un captador volumétrico de tipo Hirst. Se analizan los comportamientos estacionales e intradiarios que ha presentado cada año, así como su relación con los principales parámetros meteorológicos. También se comparan estos resultados con los obtenidos en otras ciudades españolas y europeas. Este tipo polínico se detecta en la atmósfera de Málaga fundamentalmente desde finales de Marzo hasta principios de Octubre, presentando un pico máximo en primavera (casi siempre en Abril) y otro, de menor intensidad, en verano. Las concentraciones medias diarias muy pocas veces suelen superar los 50 granos por metro cúbico de aire. Su distribucción intradiaria fue similar en todos los años estudiados, con un máximo a las 14 horas locales, que supone algo más del 15\% del polen total diario. Se han observado diferencias cuantitativas en los valores anuales, mensuales (Abril y Mayo) y máximos diarios, siendo todos ellos más bajos en 1995 y 1997, y más elevados durante 1993. Esto se ha relacionado con la cantidad de precipitaciones acumuladas durante los dos meses anteriores al periodo de polinación. Las correlaciones obtenidas entre los valores diarios de concentración atmosférica de polen y diferentes parámetros meteorológicos muestran que las fluctuaciones de concentración atmosférica de polen se asocian positivamente con las precipitaciones y la dirección del viento.
\end{abstract}

Palabras clave. Aerobiología, alergia, polen, Chenopodiaceae, Amaranthaceae, Málaga, fenología, meteorología.

\begin{abstract}
Airborne pollen of Chenopodiaceae-Amaranthaceae in Malaga (S. Spain) and its relationship with meteorological parameters. In this work we present the airborne pollen measurements of Chenopodiaceae-Amaranthaceae in Málaga during the 1992-1997 period. The study was carried out whit the aid of a volumetric spore-trap (Burkard seven-day recorder) located on the flat roof of the Faculty of Medicine, $1 \mathrm{~km}$ far from the city centre. We have also analysed the seasonal and intradiurnal variations and their relationship with the meteorological parameters by means of the Spearman's correlation test. This pollen type was mainly detected in the atmosphere of Málaga from late March to early October, with a maximum peak in Spring (April) and other smaller one during Summer. The mean daily pollen concentrations were not very high and they did not usually reach more than 50 pollen grains per cubic meter of air. The pattern of intradiurnal distribution was very uniform for all the years studied, the maximum peaks obtaining at the same hour (14:00 Spanish official Time), representing more than $15 \%$ of total daily pollen. However, we observed important differences in the quantities of total annual pollen througout the different years studied, and the maximum peaks detected, probably due to the differences in the cumulative rainfalls prior (two months before) to the pollination period. The correlations obtained between the daily pollen concentrations and the different meteorological parameters show that the presence of this pollen type in the air of Malaga is positively associated with the previous rainfall and wind direction.
\end{abstract}

Key words. Aerobiology, allergy, pollen, Chenopodiaceae, Amaranthaceae, Malaga, phenology, meteorology. 


\section{INTRODUCCIÓN}

Las familias Chenopodiaceae y Amaranthaceae se encuentran ampliamente representadas en la provincia de Málaga, donde han sido citadas numerosas especies de los géneros Arthrocnemun, Atriplex, Beta, Chenopodium, Halimione, Suaeda y Salsola (Chenopodiaceae), así como de Achyranthes, Alternanthera y Amaranthus (Amaranthaceae). Como especies ornamentales citaremos las de los géneros Kochia, Alternanthera, Celosia e Iresine (Trigo, 1992). De todas ellas, las más frecuentes son Beta vulgaris L., así como diversas especies de Amaranthus y Chenopodium. Estas especies, de forma general, crecen en lugares nitrificados, ocupando suelos ricos en sales inorgánicas, tanto del litoral como del interior, y formando parte de las comunidades ruderales, nitrófilas y halófilas. Por otra parte, numerosas especies presentan adaptaciones a la sequía, por lo que también aparecen en comunidades xerófilas.

La polinización de estas plantas es fundamentalmente anemófila, siendo sus pólenes bastante similares, por lo que forman un grupo estenopalino. Se trata de pólenes polipantoporados, apolares, radiosimétricos y circulares, de tamaño pequeño a mediano (10$50 \mu \mathrm{m}$ de diámetro). Los poros, en número de 25-60 por grano de polen, tienen de 2-3 $\mu \mathrm{m}$ de diámetro y se encuentran regularmente distribuidos por toda la superficie, que es equinulada. El polen de otras especies, como Salsola, presentan un contorno subpoligonal (Palermo et al., 1987; Crimi et al., 1988), así como Alternanthera, aunque este último tipo polínico se diferencia por presentar poros muchos más anchos $(8 \mu \mathrm{m})$ y menos numerosos (12-16).

Estas familias son consideradas por numerosos autores como causantes de polinosis, pero su importancia varía según las regiones y el taxon en cuestión (Lombardero et al., 1991). Salsola kali L., debido a que desprende grandes cantidades de polen, se ha considerado como la especie más importante dentro de este grupo desde el punto de vista alergénico en el área mediterránea (Panayotopulu et al., 1991), así como en el oeste de Estados Unidos (Lewis et al., 1983). Beta vulgaris produce rinitis en zonas donde se cultiva (de sus raíces se extrae sacarosa, y también se consumen, como verdura, sus peciolos y hojas). Por otra parte, se sugiere la posibilidad de que existan alérgenos comunes entre los géneros y especies de ambas familias, pues hay indicios de reactividad cruzada entre ellos (Lamson, 1931). Actualmente, en un estudio llevado a cabo en la provincia de Málaga, García González (1994) y Torrecillas et al. (1998) destacaron la importacia clínica del polen de ambas familias, confirmando que son la cuarta causa de polinosis en Málaga, después del olivo, las gramíneas y la Parietaria. De los 200 pacientes malagueños diagnosticados de rinoconjuntivitis y/o asma estacional, 40 (20\%) fueron sensibles a los extractos de polen de Chenopodium album, siendo dos de ellos monosensibles. Por otra parte, 34 pacientes (17\%) lo fueron a los de Salsola kali (op. cit.). Otras ciudades españolas en donde se han detectado actualmente una alta prevalencia de pruebas cutáneas positivas a estos dos pólenes fueron Elche, Toledo, Logroño, Ciudad Real y Zaragoza (Subiza et al., 1998).

Debido a que el polen de ChenopodiaceaeAmaranthaceae se detecta en la atmósfera de Málaga en cantidades importantes, ocupando entre el cuarto y séptimo lugar en orden de abundancia anual (Cabezudo et al., 1994; Recio et al., 1995; Toro et al., 1996; Trigo et al., 1998), y debido a su demostrado poder alergógeno, se ha creído conveniente realizar un estudio más profundo de este tipo polínico, analizando su comportamiento estacional e intradiario y estudiando la posible relación que podrían tener con los diferentes parámetros meteorológicos. 


\section{MATERIAL Y MÉTODOS}

El muestreo aerobiológico se ha llevado a cabo durante los años 1992-1997, ambos inclusive, mediante un captador volumétrico de tipo Hirst (1952), modelo seven-dayrecording de la casa Burkard, situado a unos 15 m del nivel del suelo, en la azotea de la Facultad de Medicina de la Universidad de Málaga. Este edifico se encuentra ubicado aproximadamente a $1 \mathrm{~km}$ al oeste del núcleo urbano y en una zona abierta que permite la libre circulación del aire.

El recuento de los granos de polen se ha realizado según el método recomendado por la Red Española de Aerobiología (Domínguez Vilches et al., 1991; Domínguez Vilches, 1995), utilizandose un objetivo de 40x y habiéndose observado 4 barridos longitudinales por preparación microscópica. Los resultados se presentan como valores medios diarios, expresados en número de granos de polen por metro cúbico de aire $\left(\mathrm{N}^{\circ}\right.$ g.p. $\left./ \mathrm{m}^{3}\right)$.

El período de polinación principal (PPP) se ha determinado en base al $95 \%$ del polen total anual, utilizando valores acumulados desde el día 1 de Enero (Pathirane, 1975) y eliminando el $2,5 \%$ inicial y final con objeto de evitar las colas de ambos extremos de la curva.

Para establecer los modelos de distribucción intradiario, se han tomando los días exentos de precipitaciones cuya concentración media diaria era igual o superior a la media del período de polinación principal. Para el cálculo del índice de distribucción intradiario (IDI) se ha seguido la metodología propuesta por Trigo et al. (1997).

El análisis de la relación existente entre los valores diarios de concentraciones polínicas y de diversos parámetros meteorológicos se ha realizado por medio del test de correlación no paramétrico de Spearman. Las variables meteorológicas utilizadas son: horas de sol, precipitaciones (en $\mathrm{mm}$ ), humedad relativa, temperaturas media, máxima y mínima $\left(\mathrm{en}^{\circ} \mathrm{C}\right)$, velocidad media del viento (en $\mathrm{km} / \mathrm{h}$ ), dirección del viento y calmas (\% de cada uno de los cuatro cuadrantes y de calmas). También se han utilizado los valores acumulados de la temperatura media, horas de sol y precipitaciones, a partir del solsticio de invierno (22 de Diciembre). Este análisis estadístico se ha efectuado, separadamente, para los dos meses de mayor concentración polínica: Abril y Mayo, siendo generalmente el sentido de la curva de concentración polínica ascendente durante el primero y descendente durante el segundo. En este caso, no se ha efectuado este análisis para los periodos pre- y postpico del PPP, debido a la forma general de la curva, con dos picos, uno de mayor intensidad en primavera y otro menor en verano.

Los datos meteorológicos han sido cedidos por el Centro Meteorológico Territorial de Andalucía Oriental, y corresponden a la Estación del Aeropuerto de Málaga, situada a unos $5 \mathrm{~km}$ al sur de la estación de muestreo.

\section{RESULTADOS}

\section{Variación estacional}

Este tipo polínico aparece en la atmósfera de Málaga durante todo el año, aunque fundamentalmente desde finales de Marzo hasta principios de Octubre (fig. 1), concentrándose durante los meses de Abril y Mayo la mayor parte del polen total anual de este taxon (fig. 1 y tab. 1), lo que supone, aproximadamente, el $62 \%$ según datos del periodo 1992-1997. La duración de la estación de polinación princiapal (PPP) ha sido relativamente similar en todos los años estudiados, con una media de 186 días y una desviación stardard de \pm 20 días (tab. 2).

Durante los años de estudio (1992-1997) la concentración total anual media de este tipo polínico fue de 1597 g.p. $/ \mathrm{m}^{3}$. Sin embargo, sólo se obtuvieron valores similares a la media durante los años 1992 y 1994. En 1995 y 1997 

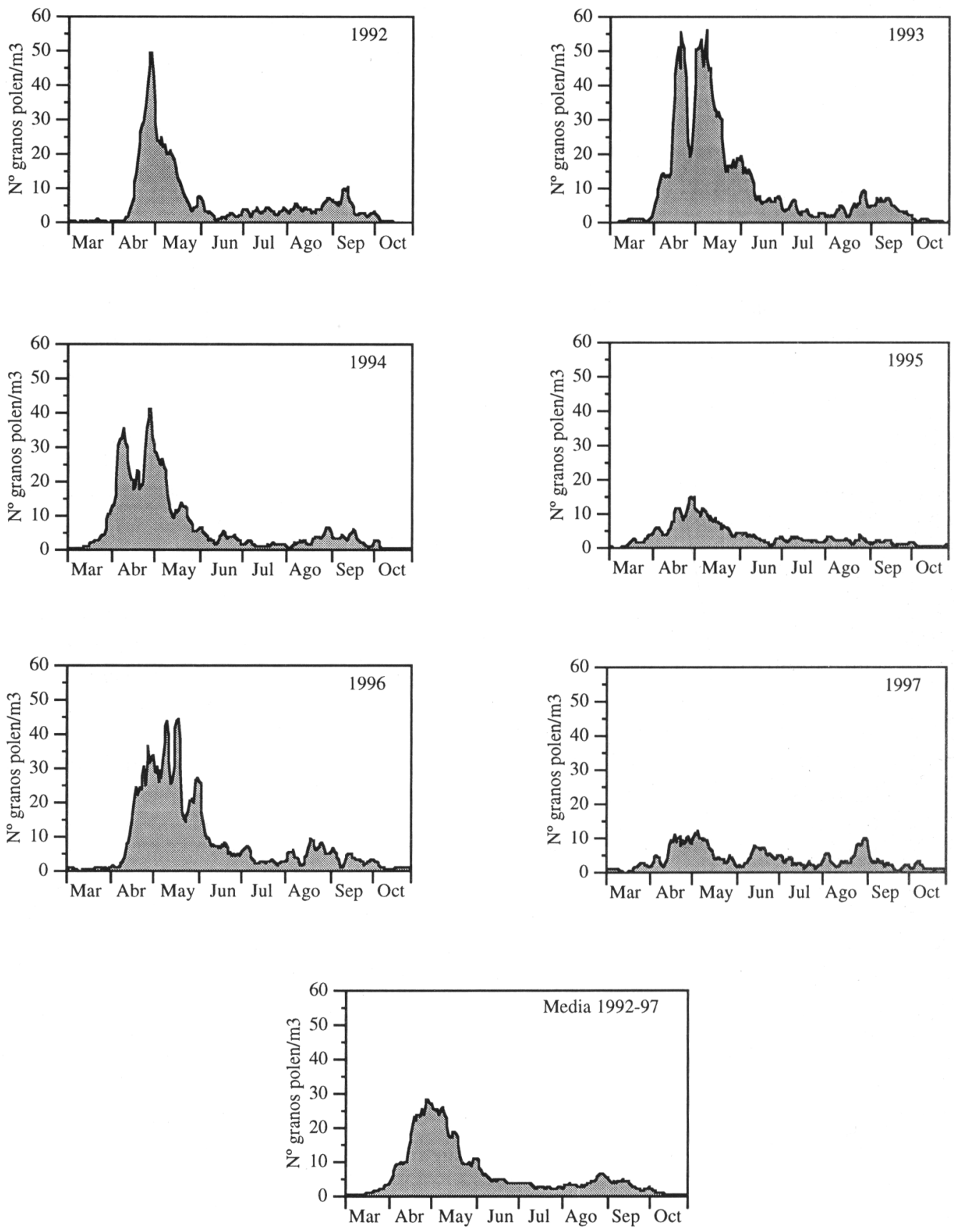

Figura 1. Medias móviles de cinco días de concentración atmosférica de polen de ChenopodiaceaeAmaranthaceae en Málaga. Five days running means for the concentration of airborne ChenopodiaceaeAmaranthaceae pollen in Malaga. 


\begin{tabular}{|c|c|c|c|c|c|c|c|c|c|c|c|c|c|c|}
\hline & $\mathrm{E}$ & $\mathrm{F}$ & $\mathrm{M}$ & A & $\mathrm{M}$ & $\mathrm{J}$ & $\mathrm{J}$ & A & $S$ & $\mathrm{O}$ & $\mathrm{N}$ & D & Total & $\%$ \\
\hline 1992 & 3 & 10 & 15 & 483 & 465 & 87 & 109 & 130 & 163 & 27 & 9 & 8 & 1507 & 6,7 \\
\hline 1993 & 5 & 8 & 15 & 755 & 1057 & 305 & 106 & 120 & 147 & 19 & 2 & 2 & 2541 & 8,0 \\
\hline 1994 & 3 & 5 & 79 & 753 & 476 & 119 & 49 & 68 & 106 & 22 & 8 & 6 & 1694 & 6,0 \\
\hline 1995 & 4 & 6 & 39 & 240 & 241 & 80 & 71 & 70 & 42 & 13 & 10 & 4 & 819 & 4,1 \\
\hline 1996 & 3 & 3 & 15 & 453 & 878 & 303 & 107 & 161 & 103 & 42 & 11 & 9 & 2087 & 4,9 \\
\hline 1997 & 4 & 9 & 32 & 189 & 206 & 133 & 83 & 119 & 97 & 43 & 7 & 11 & 934 & 1,4 \\
\hline
\end{tabular}

Tabla 1. Valores mensuales y anuales de polen atmosférico de Chenopodiaceae-Amaranthaceae (suma de concentraciones medias diarias expresadas en granos $/ \mathrm{m}^{3}$ de aire) y porcentajes anuales con respecto al polen total, registrados en Málaga durante los años 1992-97. Monyhly and annual values of airborne Chenopodiaceae-Amaranthaceae pollen (sum of the daily average concentrations expressed in grains $/ \mathrm{m}^{3}$ of air) recorded in Málaga 1992-97. The last column expresses the annual percentage represented by airborne Chenopodiaceae-Amaranthaceae pollen of the total pollen from all taxa.

estos valores anuales se situaron muy por debajo de la media (819 y 934 g.p. $/ \mathrm{m}^{3}$, respectivamente), mientras que, en 1993, fue considerablemente superior (2541 g.p. $/ \mathrm{m}^{3}$ ). Con respecto al polen total anual, el de Chenopodiaceae-Amaranthaceae representó entre el 1,43\% en 1997 y el 8,02\% en 1993.

Los valores máximos diarios de este tipo de polen se obtuvieron casi siempre en la segunda quincena del mes de Abril, exceptuando en 1996, que se retrasó hasta mediados de Mayo. Los valores de estos máximos fueron muy variables, oscilando entre los 19 g.p. $/ \mathrm{m}^{3}$ en 1997 y $\operatorname{los} 107$ g.p. $/ \mathrm{m}^{3}$ en 1993 (tab. 2). Generalmente este valor máximo no suele exceder los 100 g.p. $/ \mathrm{m}^{3}$, siendo su media de $63 \pm 32$ g.p. $/ \mathrm{m}^{3}$. Además, son muy pocos los días en los que se superan los 50 granos de polen por $\mathrm{m}^{3}$. Durante los años 1995 y 1997 en ningún caso se alcanzó este nivel de concentración polínica diaria (tab. 2).

\section{Variación intradiaria}

Durante los años de estudio las curvas de variación intradiaria (fig. 2) presentaron un comportamiento bastante estable, concentrándose la mayor parte del polen entre las 12:00 y las 16:00 horas locales (+1 UT) y registrándose el pico máximo siempre a las 14:00 h. Estos picos suponen entre el 14 y el
$22 \%$ del polen total diario. El pico máximo más elevado se obtuvo en 1993 y el más bajo en 1997, años que coinciden con el de mayor registro anual y con uno de los más bajos, respectivamente.

El índice de distribución intradiaria (IDI) medio para este taxon es de 0,26 $\pm 0,06$, correspondiendo el valor más alto al año 1993 $(0,34)$ y el más bajo a $1997(0,09)$.

\section{Correlación con parámetros meteorológicos}

En la tabla 3 se presentan los coeficientes de correlación de Spearman obtenidos entre las concentraciónes medias de polen y los diferentes parámetros meteorológicos, durante los meses de Abril y Mayo de todos los años estudiados. Se observa que el nivel de significación de estos coeficientes ha sido diferente en cada año de estudio, no obstante podemos apreciar algunas tendencias generales.

Se han obtenido correlaciones altamente significativas con las variables acumulativas de temperaturas, horas de sol y precipitaciones, que siempre fueron positivas en Abril y negativas en Mayo.

Con respecto a las temperaturas diarias, sólo han resultado significativas las correlaciones en los años 1992 y 1997, siendo también positivas en Abril y negativas en Mayo.

En cuanto a las precipitaciones, sólo se 


\begin{tabular}{|c|c|c|c|c|c|c|c|c|}
\hline & 1992 & 1993 & 1994 & 1995 & 1996 & 1997 & Media & SD \\
\hline \multirow[t]{3}{*}{ Fecha del PPP } & $13 \mathrm{Abr}$ & $6 \mathrm{Abr}$ & $26 \mathrm{Mar}$ & $18 \mathrm{Mar}$ & $14 \mathrm{Abr}$ & $20 \mathrm{Mar}$ & $1 \mathrm{Abr}$ & 12 \\
\hline & $\mathrm{a}$ & a & a & $\mathrm{a}$ & a & $\mathrm{a}$ & $\mathrm{a}$ & \\
\hline & $3 \mathrm{Oct}$ & 19 Sep & $25 \mathrm{Sep}$ & $5 \mathrm{Oct}$ & $3 \mathrm{Oct}$ & $23 \mathrm{Oct}$ & $3 \mathrm{Oct}$ & 11 \\
\hline Longitud del PPP ( $\mathrm{n}^{\circ}$ días) & 174 & 167 & 184 & 202 & 173 & 219 & 186 & 20 \\
\hline Máximo diario $\left(\right.$ granos $/ \mathrm{m}^{3}$ ) & 60 & 107 & 73 & 22 & 77 & 19 & 63 & 32,5 \\
\hline Fecha del máximo diario & $29 \mathrm{Abr}$ & $23 \mathrm{Abr}$ & $27 \mathrm{Abr}$ & $29 \mathrm{Abr}$ & 19 May & $17 \mathrm{Abr}$ & $29 \mathrm{Abr}$ & 11 \\
\hline $\mathrm{N}^{0}$ días con $<10$ granos $/ \mathrm{m}^{3}$ & 208 & 166 & 203 & 223 & 199 & 243 & 207 & 26 \\
\hline $\mathrm{N}^{\circ}$ días con $11-50$ granos $/ \mathrm{m}^{3}$ & 34 & 57 & 45 & 17 & 49 & 16 & 36 & 17 \\
\hline $\mathrm{N}^{\circ}$ días con $51-100$ granos $/ \mathrm{m}^{3}$ & 3 & 9 & 2 & 0 & 6 & 0 & 3 & 4 \\
\hline $\mathrm{N}^{\circ}$ días con $>100$ granos $/ \mathrm{m}^{3}$ & 0 & 1 & 0 & 0 & 0 & 0 & 0 & 0 \\
\hline
\end{tabular}

Tabla 2. Datos característicos para el polen de Chenopodiaceae-Amaranthaceae en Málaga durante el período de polinación principal (PPP). Characteristic data for airborne Chenopodiaceae-Amaranthaceae pollen in Málaga during the principal period of pollination (PPP): Dates of start and end of PPP; length of PPP (No. days); maximum daily pollen count (grains/ $\left.\mathrm{m}^{3}\right)$; date of maximum daily pollen count; No. of days with $<10,11-50,51-100$ and $>100$ grains $/ \mathrm{m}^{3} . \mathrm{SD}=$ standard desviation.

han obtenido algunas correlaciones significativas que fueron positivas tanto en Abril como en Mayo. Por el contrario la humedad relativa, que resultó pocas veces significativa, generalmente se asoció de forma negativa con las concentraciones de polen.

En cuanto a la dirección del viento, se ha observado que, en general, durante los periodos en que se detectaron grandes cantidades de polen se obtuvieron correlaciones significativas y positivas con el viento del segundo cuadrante y negativas con el del cuarto cuadrante, mientras que en los periodos en que las cantidades detectadas fueron más bajas sucedió lo contrario (las correlaciones significativas fueron negativas con el viento del segundo cuadrante y positivas con el del cuarto).

\section{DISCUSIÓN}

\section{Variación estacional}

El polen de ChenopodiaceaeAmaranthaceae ha presentado un comportamiento estacional bastante estable en Málaga durante todos los años de estudio, con un pico máximo en primavera y otro menor a finales de verano. Esta curva obtenida en Málaga probablemente se debe a que los pólenes que se recogen durante la primavera corresponden, en su mayoría, a chenopodiáceas y fundamentalmente a Beta vulgaris, una especie muy común en toda la vega del Guadalhorce, mientras que los de verano proceden mayoritariamente de amarantáceas.

En general, se trata de un tipo polínico muy común en toda Europa, con un período de polinación muy variable y que no suele alcanzar niveles altos en la atmósfera. En el resto de Europa normalmente los valores máximos se registran sólo en verano (Agosto-Septiembre), o bien presentan un pico de escasa intensidad a finales de primavera y otro mayor en verano, como sucede en el sur de Italia, Grecia y Cataluña, al estar comprendida dentro del área mediterránea (Spieksma, 1991; Nilsson \& Spieksma, 1992). No obstante, en Murcia (Munuera et al., 1995), Almería (Belmonte \& Roure, 1991) y Sevilla (Candau et al., 1998) se ha observado un comportamiento similar al de Málaga, detectándose las mayores concentraciones polínicas en los meses de 

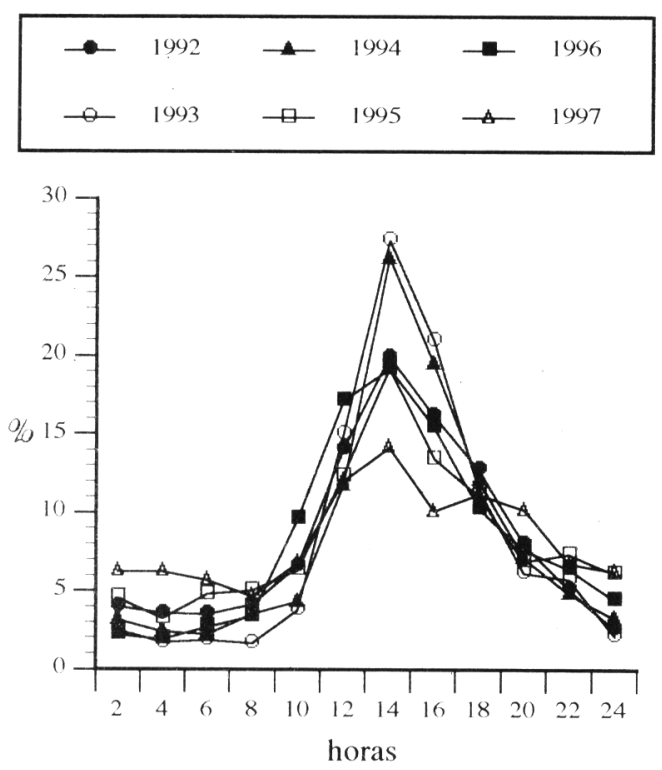

Figura 2. Variación intradiaria de polen atmosférico de Chenopodiaceae-Amaranthaceae en Málaga. Intradiurnal variation of airborne ChenopodiaceaeAmaranthaceae pollen in Malaga (Spainsh Official Time).

primavera y un pico máximo en el mes de Abril. Para el resto de las ciudades españolas la evolución de la curva anual es diferente, aunque se ha observado que en algunas localidades como Córdoba (Galán et al., 1998) y Chirivel (Cariñanos et al., 1998), el comportamiento es parecido al de Málaga si bien existe un evidente retraso en el pico de primavera (Mayo-Junio).

Los niveles aeropolínicos anuales y semanales alcanzados por este tipo de polen son similares a los obtenidos en Córdoba (Galán et al., 1998), Sevilla (Candau et al., 1998) y Badajoz (Silva et al., 1998) durante los años 1995-1996. Las estaciones que superaron a Málaga en estos niveles han sido Huelva (González Minero et al., 1998), Zaragoza (Bermejo et al., 1998) y Cartagena (MorenoGrau et al., 1998), donde el polen este taxon fue relativamente abundante durante estos años, probablemente debido a la presencia de grandes zonas de vegetagión halófila en las inmediaciones de las estaciones de muestreo.

Las diferencias interanuales detectadas en Málaga parecen indicar que las concentraciones atmosféricas de polen de Chenopodiaceae-Amaranthaceae dependen, en mayor o menor medida, de la cantidad de precipitaciones acumuladas, aproximadamente, hasta dos meses anteriores (fig. 3). Este fenómeno ha sido observado también en otros pólenes de plantas herbáceas como gramíneas (Toro et al., 1996) y Plantago (Recio et al., 1997), e incluso en uno de los pólenes de mayor incidencia en Málaga, el de olivo (Recio et al., 1996).

En relación con el resto de los táxones registrados en Málaga, el polen de Chenopodiaceae-Amaranthaceae ocupó, durante el periodo estudiado, entre el cuarto y el décimo puesto, en orden de abundancia anual.

\section{Variación intradiaria}

Son muy escasos los trabajos que muestran el comportamiento intradiario del polen de Chenopodiaceae-Amaranthaceae. Tan sólo hemos encontrado los trabajos de Käpylä (1981) para Jyväskylä (Finlandia) y de Galán et al. (1989, 1991) para Córdoba, ademas del realizado por Trigo et al. (1997) para Málaga. Según el muestreo aerobiológico realizado por Käpylä (1981) durante los meses de Junio, Julio y Agosto de 1977 y 1978, este polen presentó un comportamiento similar al obtenido en Málaga, con la diferencia de que el pico máximo estaba un poco más adelantado (12 horas) y representaba sólo poco más del $10 \%$ del total diario. Lo mismo ocurrió en Córdoba (Galán et al., 1989, 1991), aunque hubo variabilidad en los momentos de máxima concentración, que osciló entre las 12 horas en 1982 y 1984 , y 14 horas en 1983. Por todo ello, podemos afirmar que, en general, el polen de las chenopodiáceas y amarantáceas suele concentrarse en el aire, en mayor o menor 
ะี ฮั.

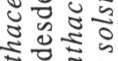

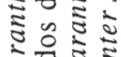

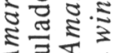

خ元

\&

: $): 0$

:응

골

U造壱

ช $\dot{0}$ 로

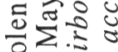

ฉर

용켱ํㅏ

둥ㅎㅎ

웡

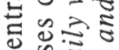

造

की शे

음으인

कै

츨 종

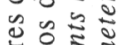

은.

을 훙

으융 원

논 $\approx \cdot 5$

可沓:

की क⿺⿻一𠃋𠃌

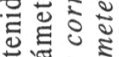

응

ำ

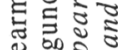

के त्र जी

䓃司

ธี है है.

르워ำ

혼 웜융 ㅇํ워ำ 递它. $\frac{0}{5}=\frac{0}{2}$ 可焉 ن की

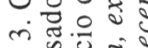
ले

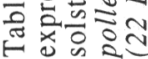


medida, en torno al mediodía-primeras horas de la tarde, cuando la insolación y las temperaturas son máximas.

Los valores del IDI alcanzados por el polen de Chenopodiaceae-Amaranthaceae son relativamente altos si se comparan con los obtenidos por otros tipos polínicos en Málaga (Trigo et al., 1997), a pesar de haberse encontrado diferencias entre un año y otro. Lo mismo podemos decir sobre la magnitud del pico de la curva gráfica.

\section{Correlación con parámetros meteorológicos}

Según los resultados de la tabla 3 y a pesar de que las significaciones de los coeficientes de correlación fueron diferentes en cada año de estudio, podemos afirmar que, de forma general, los parámetros meteorológicos que mejor explican las fluctuaciones de concentración atmosférica de polen de Chenopodiaceae-Amaranthaceae a lo largo de los días de Abril y Mayo son las precipitaciones acumuladas y la dirección del viento. La escasez de asociación obtenida con las temperaturas puede deberse a que se trata de plantas que soportan muy bien las altas temperaturas.

En cuanto a las valores acumulados de horas de sol, precipitaciones y temperaturas, el hecho de que siempre se obtuvieran correlaciones positivas durante el mes de Abril y negativas durante Mayo se debe a que la tendecia general de la curva de las concentraciones polínicas es ascendente durante Abril y descendente durante Mayo (fig. 1), al tiempo que las temperaturas presentan una curva ascendente durante ambos meses.

Por otra parte, como ya comentamos anteriormente, la figura 3 también apoya la relación positiva entre el polen y las precipitaciones de días anteriores, siendo 1995 y 1997 los años más afectados.

En cuanto a la correlación con dirección del viento, el diferente resultado obtenido entre los años de mayor y menor registro de polen, y

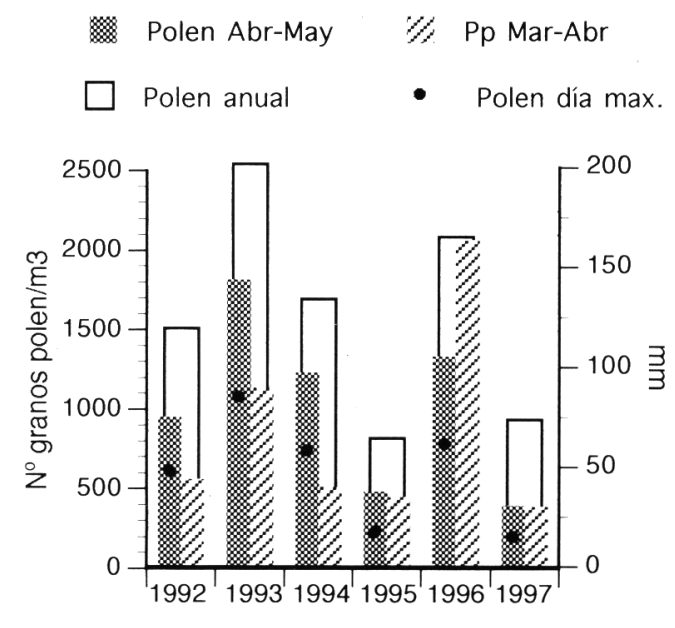

Figura 3. Relación entre las concentraciones total anual, total de los meses de mayor concentración (Abril-Mayo) y máxima diaria de polen de Chenopodiaceae-Amaranthaceae alcanzada durante los años de estudio y las precipitaciones acumuladas durante el período preestacional (Marzo-Abril). Relation between the airborne ChenopodiaceaeAmaranthaceae pollen registered during the years studied (total annual, total during the months of higher concentrations -April and May-and maximum daily concentration) and the total rainfall during the pre-season period (March-April).

sus comportamientos inversos para los meses de Abril y Mayo, está relacionado con la mayor o menor cantidad de plantas que se encuentran en flor en las zonas proximas al captador. Cuando el año es bueno en lluvias, se captura antes (Abril) el polen de las plantas más cercanas al captador, pues son las que florecen más tempranamente, mientras que en Mayo posiblemente los pólenes provengan de zonas más interiores, a través del pasillo del Guadalhorce, donde la floración es más tardía por su mayor altitud y latitud.

\section{CONCLUSIONES}

El polen de ChenopodiaceaeAmaranthaceae se detecta en la atmósfera de 
Málaga fundamentalmente desde finales de Marzo hasta principios de Octubre, presentando un pico máximo en primavera (casi siempre Abril) y otro menor en verano.

Las concentraciones medias diarias de polen de estas dos familias en Málaga no suelen superar los 100 granos por metro cúbico de aire, siendo además muy pocos los días en que se alcanzan niveles superiores a los 50 granos por metro cúbico.

La distribución intradiaria de este tipo polínico es muy estable para Málaga en cuanto a distribucción en el tiempo y momento de máxima concentración (14 horas locales).

Las cantidades totales anuales y de los meses de mayor concentración (Abril y Mayo), así como los valores máximos diarios, varían de un año a otro en función de las precipitaciones acumuladas hasta dos meses anteriores a su polinación. En Málaga los valores más bajos se obtuvieron en 1995 y 1997, y los más altos en 1993.

Los parámetros meteorológicos que mejor explican las fluctuaciones de concentración atmosférica de polen de ChenopodiaceaeAmaranthaceae de Málaga a lo largo de los días de Abril y Mayo son las precipitaciones y la dirección del viento.

Al tratarse de un taxon con demostrado poder alergizante y con una elavada incidencia en la atmósfera de Málaga, creemos que sus especies deben ser tenidas en cuenta a la hora de determinar posibles causas de polinosis, especialmente Beta vulgaris, especie que produce el pico máximo en Abril.

AGRADECIMIENTOS. Los autores desean agradecer al Ministerio de Educación y Cultura la financiación de los proyectos PB92-0814-02 y AMB97-0457-C07-05, y a las Consejerías de Salud y Educación de la Junta de Andalucía la colaboración prestada a la Red Andaluza de Aerobiología (RAA) para el desarrollo de este trabajo.

\section{BIBLIOGRAFÍA}

BELMONTE, J. \& J. ROURE - 1991- Characteristics of aeropollen dynamics at several localities in Spain. Grana 30: 364-372.

BERMEJO, D., A. M. GARCÍA, P. SANCHO y C. VALERO -1998-Aerobiología en Aragón: estación de Zaragoza (1995-1996). REA 3: 45-48.

CABEZUDO, B., M. M. TRIGO, M. RECIO y F. J. TORO -1994-Contenido polínico de la atmósfera de Málaga: Años 1992 y 1993. Acta Bot. Malacitana 19: 137-144.

CANDAU, P., C. TOMÁS, F. J. GONZÁlEZ MINERO, J. MORALES y A. M. PÉREZ TELLO -1998- Aerobiología en Andalucía: estación de Sevilla (1995-1996). REA 3: 41-44.

CARIÑANOS, P., C. GALÁN, P. ALCÁZAR y E. DOMÍNGUEZ - 1998- Aerobiología en Andalucía: estación de Chirivel (1995-1996). REA 3: 9-12.

CRIMI, N., B. PALERMO, F. PALERMO, M. P. PISTORIO, S. RIZZA, A. MISTRETTA, W. DE LEONARDIS \& N. LONGHITANO -1988- On the pollen morphology and frequenze of allergic sensitization in Sicily of the genus Salsola L. (Chenopodiaceae). Allergol. et Immunopathol. 16(4): 259-262.

DOMÍNGUEZ VILCHES, E. -1995- La Red Española de Aerobiología (REA). REA 1: 3-7.

DOMÍNGUEZ VILCHES, E., C. GALÁN, F VILLAMANDOS \& F. INFANTE-1991-Handling and evaluation of the data from the aerobiological sampling. Monografía REA/EAN 1: 1-18.

GALÁN, C., F. INFANTE, E. RUIZ DE CLAVIJO, F. GUERRA, R. MIGUEL \& E. DOMÍNGUEZ 1989-Allergy to pollen grains from Amaranthaceae and Chenopodiaceae in Cordoba, Spain. Annual and daily variation of pollen concentration. Ann. Allergy 63(11): 435-438.

GALÁN, C. R. TORMO, J. CUEVAS, F. INFANTE \& E. DOMÍNGUEZ -1991- Theoretical daily variation patterns of airborne pollen in the South-West of Spain. Grana 30: 201-209.

GALÁN, C., P. CARIÑANOS, P. ALCÁZAR, F. INFANTE, A. CASTRO y E. DOMÍNGUEZ 1998- Aerobiología en Andalucía: estación de Córdoba (1995-1996). REA 3: 13-16.

GARCÍA GONZÁLEZ, J. J. -1994-Calendario polínico de la ciudad de Málaga. Prevalencia de tests cutáneos. Tesis Doctoral. Departamento de Bioquímica y Biología Molecular. Facultad 
de Medicina. Universidad de Málaga.

GONZÁLEZ MINERO, F. J., J. MORALES, P. CANDAU, C. TOMÁS y A. M. PÉREZ TELLO -1998- Aerobiología en Andalucía: estación de Huelva (1995-1996). REA 3: 25-28.

HIRST, J. M. -1952- An automatic volumetric spore trap. Ann. Appl. Biol. 39(2): 257-265.

KÄPYLÄ, M. - 1981- Diurnal variation of non-arboreal pollen in the air in Finland. Grana 20: 55-59.

LAMSON, R. W. \& A. WATRY -1931- The importance of the Chenopodiaceae in pollinosis: with special reference to Winslow and Holbrook, Arizona. J. Allergy 4: 255-281.

LEWIS, W. H., P. VINAY \& V. E. ZENGER -1983Airborne and allergenic pollen of North America. Hopkins, London.

LOMBARDERO, M., O. DUFFORT \& J. CARREIRA 1991- Allergenic significance of chenopod pollen. In: G. D'AMATO, F. TH. M. SPIEKSMA \& S. BONINI (eds.) Allergenic Pollen and Pollinosis in Europe, pp. 128-131. Blackwell Sientific Publications, Oxford.

MORENO-GRAU, S., B. ELVIRA-RENDUELES, J. M. ANGOSTO, J. BAYO, J. MORENO y J. MORENOCLAVEL - 1998- Aerobiología en Murcia: estación de Cartagena (1995-1996). REA 3: 89-92.

MUUNUERA, M., J. S. CARRION \& J. GUERRA 1995- Approaches to airborne pollen in SE Spain. First survey in Murcia: one year of pollen monitoring (1993.94). Aerobiologia 11: 189-194.

NILSSON, S. \& F. TH. M. SPIEKSMA (eds.) 1992- Traveller's Allergy Service Guide. Swedish Museum of Natural History and Fisons Sweden AB, Sweden.

PALERMO, B., N. CRIMI, F. PALERMO, M. P. PISTORIO, S. RIZZA, A. MISTRETTA, C. DI MARCO, A. ZIZZA \& N. LONGHITANO -1987Salsola: caratterizzazione morfopalinologica e incidenza di sensibilizzazione allergica in Sicilia. Aerobiologia 3: 31-36.

PANAYOTOPULU, E., L. CENCI \& A. TURSI 1991- Salsola kali: a new etiology of pollinosis in the Mediterranean area of Apulia -Italy. Grana 30: 147-149.

PATHIRANE, L. - 1975- Graphical determination of the main pollen season. Pollen et Spores 17(4): 609-610.

RECIO, M., M. M. TRIGO, F. J. TORO y B. CABEZUDO -1995- Contenido polínico de la atmósfera de Málaga: Año 1994. Acta Bot. Malacitana 20: 83-90.

RECIO, M., B. CABEZUDO, M. M. TRIGO \& F. J.
TORO -1996- Olea europaea pollen in the atmosphere of Málaga (S. Spain) and its relationship with meteorological parameters. Grana 35: 308-313.

RECIO, M. M. M. TRIGO, F. J. TORO y B. CABEZUDO -1997- Incidencia del polen de Plantago en la atmósfera de Málaga y su relación con los parámetros meteorológicos. Acta Bot. Malacitana 22: 103-113.

SILVA, Y., A. F. MUÑOZ, R. TORMO y D. RECIO -1998- Aerobiología en Extremadura: estación de Badajoz (1995-1996). REA 3: 61-64.

SPIEKSMA, F. TH. M. -1991- Regional European Pollen Calendars In: G. D'AMATO, F. TH. M. SPIEKSMA \& S. BONINI (eds.) Allergenic Pollen and Pollinosis in Europe, pp. 49-65. Blackwell Sientific Publications, Oxford.

SUBIZA, J., F. FEO BRITO, J. POLA, J. FERNÁNDEZ, M. JEREZ y M. FERREIRO -1998- Pólenes alergénicos y polinosis en 12 ciudades españolas. Rev. Esp. Alergol. Inmunol. Clín. 13(2): 45-58.

TORO, F. J., M. RECIO, M. M. TRIGO y B. CABEZUDO -1996- Contenido polínico de la atmósfera de Málaga: Año 1995. Acta Bot. Malacitana 21: 57-63.

TORRECILLAS, M., J. J. GARCÍA GONZÁLEZ, M. T. PALOMEQUE, C. MUÑOZ, J. M. BARCELÓ, J. L. DE LA FUENTE, J. M. VEGA CHICOTE y A. MIRANDA - 1998-Prevalencia de sensibilizaciones en pacientes con polinosis de la provinica de Málaga. Rev. Esp. Alergol. Inmunol. Clín. 13(2): 122-125.

TRIGO, M. M. -1992- Flora ornamental y jardines de Málaga. Contribucción al estudio polínico de especies ornamentales con interés alergógeno. Universidad de Málaga. Colección Tesis doctorales 71, Málaga.

TRIGO, M. M., M. RECIO, F. J. TORO \& B. CABEZUDO - 1997- Intradiurnal fluctuations in airborne pollen in Málaga (S. Spain): a quantitative method. Grana 36: 39-43.

TRIGO, M. M., M. RECIO, F. J. TORO \& B. CABEZUDO - 1998- Aerobiología en Andalucía: estación de Málaga (1995-1996). REA 3: 33-36.

Aceptado para su publicación en Septiembre de 1998

Dirección de los autores. Dpto. Biología Vegetal. Fac. Ciencias. Universidad de Málaga. Apdo. 59. 29080, Málaga, Tlfno. 95 2131912. E-mail: aerox@uma.es 